\title{
Construction and Application of Economic and Management Experimental Teaching Platform Based on Cloud Desktop
}

\author{
Huang Xuejian ${ }^{a}$, Wang Gensheng ${ }^{b}$, Hu Xiangliangc, Wu Xiaofang ${ }^{\mathrm{d}}$ \\ Computer Practice Teaching Center, Jiangxi University of Finance and Economics, Nanchang, 330013, China

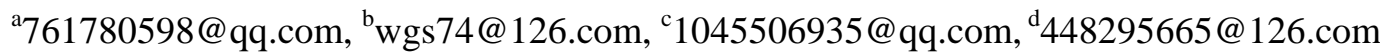

Keywords: Economic Management; Experimental Teaching Platform; Cloud Desktop; Cloud Computing.

\begin{abstract}
As an important means of training practical talents, experimental teaching is attracting more and more attention from universities. In view of the problems of high cost, difficult maintenance, low utilization rate and difficult sharing in the experimental teaching platform of the economic management class, this paper puts forward the application of cloud desktop technology to the construction of the experimental teaching platform. The advantages and structure of the platform are analyzed in detail, and combined with the case of the application of Jiangxi University of Finance and Economics, it is found that the platform not only improves the level of experimental teaching experience, but also greatly facilitates the management and maintenance of the laboratory, and effectively promotes the information construction level of the experimental teaching platform.
\end{abstract}

\section{Introduction}

With the popularization of higher education, many colleges and universities regard the training of applied talents as the training objectives of economics and management majors. In order to cultivate practical talents with practical ability, innovation ability, coordination ability and communication ability, experimental teaching has become an important part of [1]. The experiment of economic management has the characteristics of cross professional, multi role and multidisciplinary. It is an important task for many colleges and universities to build a complete set of experimental teaching platform for economic management.

\section{The Problems in the Traditional Experimental Teaching Platform}

At present, the experimental teaching platform of economics and management is mainly based on the laboratory and its hardware and software environment. With the development of education information and the attention of colleges and universities to the training of students' practical ability, the construction of the economics and management laboratory in many colleges and universities has made some achievements [2]. However, there are still the following general problems:

\subsection{High Cost of Laboratory Construction}

The construction of laboratory mainly focuses on two aspects: software and hardware. The traditional CS model experiment teaching software is used in local installation, and there are many kinds of software that need to be installed. So there is a lot of requirements for the configuration of the computer, so the cost of the hardware should be invested in the construction. And with the development of software, the performance of computer hardware will not keep up with the operating system and soft. Basically, every 5 years or so, it is necessary to update and eliminate a number of machines. In terms of software, due to the development of technology and discipline, teaching software must be constantly updated and upgraded. 


\subsection{The Workload of Laboratory Management and Maintenance is Great}

Because of the different requirements for the software environment of different experimental courses, in order to cope with this situation, the laboratory managers usually need to divide the computer into multiple partitions, install different system environment for each partition, and install the corresponding experiment course software in different system environment. Since the experimental course arrangements are different for each semester, the lab managers need to deploy the environment ahead of time and carry out the related tests, which usually takes a few days before they start the semester.

\subsection{Laboratory Opening Time and Site Limitation}

Due to the limitation of management means, the traditional laboratory is difficult to release at any time and share it with teachers and students, so that laboratory resources can't be fully utilized. And because of the fixed place of experiment, students and teachers can't carry out experiments outside the laboratory.

\subsection{The Experimental Data can't be Preserved for a long time}

In order to prevent system damage and virus infection, laboratory administrators generally use a restore card or hard disk protection card to manage the system, so that every computer restarts back to the most original state of protection. This causes the local experimental data to be deleted after each restart. Users need to manually copy data after each experiment.

\section{Cloud Desktop}

Cloud desktop is a typical application of cloud computing technology, enabling users to connect to cloud computing resources through thin clients. Users manipulate the hardware and software resources on a remote server through a virtual desktop, and all operating systems and application software used by the user are running on the remote cloud server [3].

The cloud desktop can make different personalized cloud desktop environments according to the software and hardware environment requirements of different classes of experimental courses. Users only need to log on to the corresponding cloud desktop through a simple thin client. The use of cloud desktop has brought many advantages to the construction and management of the laboratory of economics and management.

\subsection{Reducing the cost of Laboratory Construction}

The performance requirements of the terminal devices used by the cloud desktop are not high, and the ordinary thin clients, mobile terminals and ordinary PC are all satisfied, and the compatibility is very good, and they do not need to be repeatedly invested in these hardware. Cloud server resources support dynamic expansion, when the configuration can't meet the requirements of service, by upgrading or adding new cloud servers to meet

\subsection{Improve the Efficiency of Management}

Administrators can finish maintenance and update of all cloud desktop through the background server. According to the requirements of experimental teaching, the system and software are installed on the master machine through the management machine, and then all the cloud desktop is deployed in a unified way. It does not need to be deployed separately to different models, different systems and different configurations of computers, which greatly improves the efficiency of management.

\subsection{Breaking the Limits of Time and Space}

The use of cloud desktop can be connected to any ordinary terminal device, such as ordinary PC, iPad, mobile phone and so on. Users do not need to experiment in fixed places and fixed time. In the network environment, they can be connected to their experimental environment (operating system, application software and experimental data) in the network environment, and carry out the related 
experiments anytime and anywhere.

\subsection{Improving the Stability of Experimental Teaching}

Traditional experimental teaching methods often fail to carry out normal operations because of system failure, crash, server failure and other reasons. The cloud desktop takes the system and the operation of the software through the cloud server. The local connection terminal is only a virtual control interface. All the operation and data are stored on the cloud server. The user does not have to worry about the collapse of the terminal and results in the loss of the data.

\section{Design of experimental platform for economics and management based on cloud desktop}

Using cloud computing technology, such as remote control technology and server virtualization technology, to design the platform of economics and management experimental teaching platform. The specific platform architecture, as shown in Fig 1, is divided into 6 levels from the bottom to the first, in turn, the physical resource layer, the virtualization layer, the virtual resource scheduling layer, the application layer, the experimental platform layer, and the access terminal.

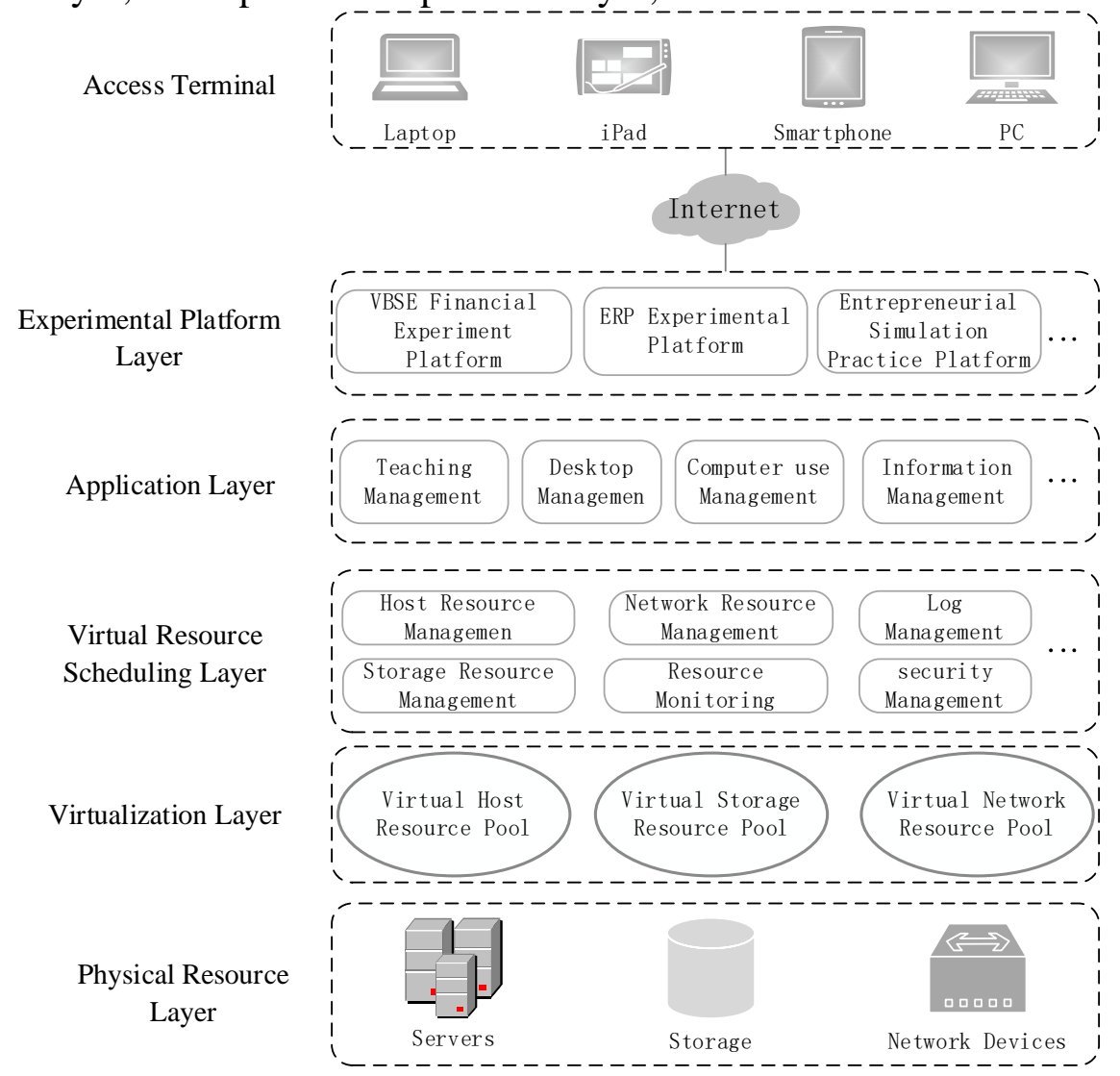

Fig 1 The architecture of experimental teaching platform based on cloud desktop

\subsection{Physical Resource Layer}

The physical resource layer is composed of server matrix, distributed storage, and network equipment to form the basic hardware resource of the cloud desktop [4], which is easy to expand, and can easily expand the capacity of hardware resources.

\subsection{Virtualization Layer}

The role of the virtualization layer is to virtualize the hardware resources of the physical layer into a manageable logical resource [5], to allocate and dispatch the physical layer resources, including the virtual host resource pool, the virtual storage resource pool and the virtual network resource pool. 


\subsection{Virtual Resource Scheduling Layer}

Allocate and schedule the host resources, storage resources and network resources after virtualization. Monitor the usage of resources, manage the security and log of the system.

\subsection{Application Layer}

The application layer is the core of the business of the whole platform. The technology of resource scheduling layer and virtualization layer are both open source and unified standards, and the application layer needs us to implement according to the specific experimental teaching business. This layer mainly includes teaching management, desktop management, computer usage management, information management and so on.

\subsection{Experimental Platform Layer}

According to the teaching needs of different experimental courses, the configuration of virtual host is carried out, then the system mirror template is installed on the virtual host, and then the related experiment course application software is installed. Finally, the unified deployment is carried out to generate the corresponding experimental teaching platform

\subsection{Access Terminal}

Users can login to the cloud desktop of related experimental platform through laptop, iPad, smartphone, PC and other terminals anytime and anywhere.

\section{Application case of economic and management experimental teaching platform based on cloud desktop}

In 2017, Jiangxi University of Finance and Economics and Guangzhou Vinzor jointly built a cloud platform for economic management experiments. This platform joins information enterprise, virtual enterprise operation simulation, virtual business social environment, innovative finance experiment and training level into platform construction. It covers many fields of experimental teaching, such as supply chain management, internal operation and management, business management, business environment simulation, collaborative office, etc. On the whole, the platform is a comprehensive experimental platform covering a number of knowledge fields, and can realize the pipeline training for students from enrollment to graduation. Realize the cloud desktop outside the campus to achieve multi campus resource sharing. Through the use of one semester, we have achieved good results in experimental teaching, and the feedback from teachers and students is very good. For experimental managers, they also reduced their heavy workload.

\section{Conclusions}

In the era of deep integration of information technology and education, the application of cloud computing in the education industry is also increasingly widespread. Facing the problems of high cost, heavy workload, low utilization rate and difficult sharing of traditional experimental teaching platform, development of efficient, advanced and high-quality experimental teaching platform has become an important module of information construction in universities. As a typical application of cloud computing, cloud desktop is an effective way to solve the problems of traditional economics and management experimental teaching platform. This paper analyzes the framework and advantages of constructing the experimental teaching platform of economics and management based on cloud desktop technology, and combines with the application cases of Jiangxi University of Finance and Economics. It is found that the platform not only improves the experience level of the experimental teaching, but also greatly facilitates the management and maintenance of the laboratory, and effectively promotes the level of the information construction of the experimental teaching platform. 


\section{References}

[1] Jian Yu, Jie Gao, Mankun Zhao, et al. Research and application of cloud platform in university computer laboratory[J].Laboratory Science.2018,21 (2): 94-96. (In Chinese)

[2] Xuejun Fang. Application of cloud technology in the construction of economics and Management Laboratory [J]. Inner Mongolia Science Technology \& Economy, 2016, 6(1):81-82. (In Chinese)

[3] Miaoli Wu, Xiaoying Duan. The Construction of Virtual Laboratory by Cloud Computing and the Resource Optimization [J]. China Modern Educational Equipment, 2016, 23(1), 10-12. (In Chinese)

[4] Yanzhao Wang. Exploration of experimental teaching in universities under the environment of cloud computing [J]. Laboratory Science, 2016, 19(5):102-105. (In Chinese)

[5] Lei Ge,Jianjun Wu. Research and Exploration of Colleges Cloud Platform Construction [J]. Software Engineering, 2016,19(1):50-52. (In Chinese) 